\title{
Auditory Learning: A Developmental Method
}

\author{
Yilu Zhang, Member, IEEE, Juyang Weng, Member, IEEE, and Wey-Shiuan Hwang
}

\begin{abstract}
Motivated by the human autonomous development process from infancy to adulthood, we have built a robot that develops its cognitive and behavioral skills through real-time interactions with the environment. We call such a robot a developmental robot. In this paper, we present the theory and the architecture to implement a developmental robot and discuss the related techniques that address an array of challenging technical issues. As an application, experimental results on a real robot, self-organizing, autonomous, incremental learner (SAIL), are presented with emphasis on its audition perception and audition-related action generation. In particular, the SAIL robot conducts the auditory learning from unsegmented and unlabeled speech streams without any prior knowledge about the auditory signals, such as the designated language or the phoneme models. Neither available before learning starts are the actions that the robot is expected to perform. SAIL learns the auditory commands and the desired actions from physical contacts with the environment including the trainers.
\end{abstract}

Index Terms-Classification, developmental learning, online auditory learning, principal component analysis (PCA), regression, reinforcement learning.

\section{INTRODUCTION}

W HILE speech recognition (SR) and natural language understanding (NLU) techniques have achieved great progress lately, good performance is typically reported in speaker dependent cases and/or under controlled acoustic environment [1]. There are still great difficulties in building a speech processing system that is capable of natural human-computer interactions. These difficulties have started to pose questions on the methodology adopted in machine speech learning areas [2], [3]. At the same time, a new line of research interest has emerged to explore and imitate the human cognitive development process [4], [5].

Examining the state-of-the-art methodology of machine speech learning, we can see several limitations. First, current speech processing systems usually do not have a mechanism to dynamically create new acoustic or language models. Instead, the models need to be handcrafted in advance, which makes it a rather static development procedure and prevents the system from dealing with the wide variety of environments [6]. Second,

Manuscript received April 29, 2002; revised November 21, 2004. The work was supported in part by the National Science Foundation under Grant IIS 9815191, in part by the Defense Advanced Research Projects Agency (DARPA) Electronic Technology Office under Contract DAAN02-98-C-4025, and in part by DARPA Information Technology Office under Grant DABT63-99-1-0014.

Y. Zhang is with the Research Center, General Motors Corporation, Warren, MI 48090 USA.

J. Weng is with the Department of Computer Science and Engineering, Michigan State University, East Lansing, MI 48824 USA (e-mail: weng@cse.msu.edu).

W.-S. Hwang is with the Rudolph Technologies, Inc., Flanders, NJ 07836 USA.

Digital Object Identifier 10.1109/TNN.2005.845217 to build a speech processing system, a large amount of data (speech data for an SR system or text data for an NLU system) must be collected, edited, and manually transcribed, ${ }^{1}$ which is a labor-intensive procedure and requires specific expertise. Third, the data preparation procedure discards a lot of valuable context information. Speech is an information exchange tool. Humans communicating with each other using speech typically have a common information context, which either exists in the environment or is kept in the minds of the participants. Without using the context information, an artificial speech processing system will not have a performance even close to a human being.

In general, we are interested in building a robot that develops its audition-related cognitive and behavioral skills through online and real-time interactions with the environment. Why do we incorporate a robot in speech learning? It is motivated by the discoveries in human cognitive development and animal learning, which suggests the following two features of cognitive development. 1) Autonomy. The mental activities of human children and young animals are largely autonomous, even though human children are usually under adult care. Teachers do not have access to the internal "wires" of the brain during teaching. Jean Piaget, a well-known psychologist, reported numerous observations to support his assimilation-accommodation model on how children incorporated new experiences into their existing schemata [7]. Later studies show that the time at which children acquire some concepts of the physical world significantly earlier than Piaget reported [8], [9]. In animal learning, a classical experiment by Held and Hein [10], "kitten carousel," demonstrated self-generated actions were crucial for cognitive development. Similar experimental results were reported on human infant subjects too [11]. 2) Grounding. Human children and young animals develop their knowledge about the physical world through continuous interactions using their sensory and motor organs. This intimate use of experiences from the physical world is called grounding. Studies over cats, monkeys, and human infants showed that richness of early sensory experiences could significantly affect the subjects' perceptual competence and intellectual performance ([12] on cats' edge perception, [13] on monkeys' visual cortex response to the temporarily occluded eyes, [14] on human infants growing in a foundling home and in a nursing home attached to a woman's prison). Autonomy and grounding imply that, in addition to performing physical tasks, a body is extremely important for cognitive development of a biological agent. By the same token, a robot body with a closed brain (not directly accessible to teachers), multimodal sensors, and effectors is necessary for a robot to duplicate the powerful

\footnotetext{
${ }^{1}$ It is called transcription to manually segment the speech data and translate them into strings of symbols representing the corresponding linguistic units before using them to train acoustic models.
} 
SR and understanding capabilities of human beings. Further, from an engineering point of view, an embodied artificial agent can learn in right physical contexts that are characterized by the sensory inputs from the multiple modalities. Therefore, the context information will potentially be taken into account for decision-making by the agent, which greatly facilitates speech processing.

Our goal is to explore the possibility of emulating the human learning process to some degree, which may boost the auditory capability of a machine. We follow an autonomous mental development paradigm, whose importance was discussed in a recent article published in Science [3], to build an online auditory learning robot. The autonomous mental development paradigm can be characterized as follows.

1) Body design: A human designer designs a robot body (the sensors, the effectors and the computational resources) according to the general ecological condition in which the robot will work (e.g., on land or underwater).

2) Developmental program design: A human programmer designs a task-nonspecific developmental program for the robot.

3) Mind development: The robot starts to run the developmental program and develops its mental skills through online real-time interactions with the environment, which includes humans.

A robot constructed following this autonomous development paradigm is called a developmental robot. The important point here is that robot tasks are not known or cannot be predetermined at robot design time. For example, in auditory learning, the voice commands and even the languages are not determined at design time. Therefore, there is no way to program taskspecific or environment-specific information into this new kind of program, called a developmental program. A developmental robot must generate internal representations and architecture autonomously according to the experiences it encounters. The task-nonspecific requirement is meant to push us and lead us to build a real autonomous learning agent with a powerful environment-adaptive capability.

In this paper, we report our work on audition-related learning by our developmental robot, self-organizing, autonomous, incremental learner (SAIL). Since our approach generates representation online without a need to hand design a particular model, the method potentially can be applied to any auditory inputs, such as speech, music and songs. However, we concentrate our experiments on short-phrase voice commands in the following as an example of applications.

\section{A. Developmental Robots}

Early prototypes of developmental robots include the SAIL robot at Michigan State University, East Lansing, MI [15], the Darwin V robot at the Neurosciences Institute, San Diego, CA[16], and the Illy robot at the University of Illinois, Urbana-Champaign [17].

The SAIL robot was designed as an engineering testbed for developmental programs. The SAIL-2 developmental program has been tested for autonomous derivation of representations through online, real-time development of association 1) between visual stimuli of objects and eye aiming for the objects (object evoked visual attention); 2) between visual stimuli of objects and arm prereaching for the object (vision evoked object reaching); 3) between voice stimuli and arm actions (verbal command learning and execution); and 4) between visual stimuli and locomotion effectors (vision-guided navigation). Ongoing investigations include temporal signal processing, more sophisticated attention mechanisms, and value system development [15].

The Darwin V robot by Almassy, Edelman, and Sporns was designed to provide a concrete example to show how the computational weights of neural circuits were determined by the behavioral and environmental interactions of an autonomous device [16]. Darwin V had a group of predesigned behaviors and sensory patterns. Its visual capability was implemented by blob-pattern and stripe-pattern detectors. Darwin V has been tested for developing behaviors in response to visual stimuli at different positions and orientations (visual invariance learning). It also has been tested for the association of aversive and appetitive stimuli with visual stimuli (value learning). This work is concentrated on the development of high level plasticity of the association between stimuli and behaviors.

The Illy robot project by Levinson, Huang, and colleagues focused on spoken language acquisition. The paper [17] reported how to teach the Illy robot to act according to short voice commands in different languages in real time, using entropy-guided LVQ and memory association. This work paralleled our work of auditory learning by the SAIL robot. Since the learning system of the Illy robot has a flat representation, scaling is a major concern when the learning goes on for a long time. In the SAIL robot project, various techniques, such as incremental principal component analysis (IPCA) and hierarchical discriminant analysis, are developed to tackle the scaling problem. In addition, the speech acquisition system of the Illy robot had some preprogrammed modules such as "end point detection," while the method reported in this paper does not use such modules to reach a fully developmental robot.

Another interesting work was done by Roy, Schiele, and Pentland in Media Lab at MIT [18], [19]. They developed a multimodal system which learned shape categories and their corresponding spoken names from an input consisting of naturally spoken utterances paired with visual representations of objects. Mutual information was used to extract the audio-visual lexicon from the sensory inputs. This work was motivated by the study of infant cognition and aimed at enabling a robot to learn semantic representations through sensorimotor experiences. This was a step toward the challenging real-time multimodal learning. However, the learning process was not done strictly through real-time interactions between the agent and the environment in the reported experiment. Instead, the images were manually aligned with the auditory signals before the sensory inputs were fed to the learning system.

\section{TECHNICAL ISSUES}

The strength of an autonomous audition development system lies in the elimination of explicit design of internal representation 
by human, and in enabling the system to learn directly from its real-time multimodal sensory experiences in the physical world, which is similar to the autonomous animal learning. However, this new capability raises a series of new challenges that a traditional speech learning system does not have to deal with. In the work presented here, we address the following challenges.

\section{1) Automatic Generation of Internal Representa-} tion: Internal representation includes, among others, features extracted from perceived signals, subspaces represented by clusters in sensory inputs, inter-connections between nodes, and states consisting of short-history information. For a traditional learning robot, the representation of a task is typically designed by a human programmer manually. The programmer takes the advantage of knowing the task and the environment by programming various constraints into the robot program. For the developmental robot we are interested in, no task-specific information is available until the system has been built, programming has been finished, and the robot has entered the user stage. In the user stage, the users are also the trainers of the system but they can not conduct any internal-data-level intervention that the human programmers can do. Interactions between the robot and its environment, including the users, are only through the robot's sensors and effectors. Therefore, a developmental robot must collect data and generate internal representations automatically.

2) Unsegmented Sensory Stream: The environment is sensed by a robot as continuous and endless data streams, e.g., the auditory stream from a microphone, visual streams from cameras, and tactile streams from touch sensors. To associate sensory inputs with robot actions, an important issue is to decide the appropriate contexts. For example, meaningful speech units (phonemes, words, phrases, and sentences) have varying temporal length. Without relying on a human designer to segment or transcribe sensory inputs, a robot must automatically associate the appropriate contexts with the desired actions. The temporal closeness between the sensory inputs and the actions is of essential importance for sensorimotor association. In addition to this, another thing that affects the association establishment is the feedback the robot receives from the environment, such as the reward supplied by human trainers (encouraging or discouraging signals) or nonhuman environment (collisions). However, a reward is typically delayed and is often inconsistent in the delivery time.

3) Learning Internal Behaviors: Autonomous learning is not effective without developing internal behaviors. By internal behaviors, we mean the perception-invoked actions that are applied to internal effectors, such as attention effectors, action release effectors, etc. For example, closely related to the issue of unsegmented sensory stream is the need of a selective attention behavior that allows a robot to focus on the part of the input data critical in the current situation. Another example of internal behaviors is the manipulation of internal states. Since internal behaviors are not accessible to the world outside the robot, it is very challenging to enable the robot to develop desired internal behaviors through external interactions via its sensors.

4) One-Instance Learning Without Local Minima: Online learning implies that the learning algorithm must be very adap- tive while highly reliable, which are two conflicting criteria. A system must be able to learn from as few as a single instance for association yet without getting stuck into local minima. The methods such as hidden Markov models (HMMs) typically need a preprocessing stage to estimate the initial observation probabilities and transition probabilities before the learning algorithm (e.g., Baum-Welch algorithm) starts. This is because the Baum-Welch algorithm does not give a good solution starting from a random initial guess. However, an online learning algorithm must not give a bad solution even without a preprocessing stage.

There are still other technical issues, which are beyond the scope of the work reported here, for example, the development of a high-level value system to efficiently and effectively evaluate robot behavior. Because of these new challenges, at current research stage of autonomous audition development, we should not expect the system performance to reach the level of a traditional speech learning system immediately, in either vocabulary size, speaker variation or recognition rate. However, as discussed in [3] and [5], the autonomous audition development provides new dimensions for speech learning researchers to address current difficulties in dealing with speaker variation, environmental noise, and inherent ambiguity in audition without other multimodal sensory inputs.

\section{SYSTEM ARCHITECTURE AND LEARNING STRATEGY}

In this section, we present a novel agent model and an architecture of our developmental robot to implement this model. We discuss the new techniques employed in its major components thereafter.

Definition 1: The history at time $t$ is defined as $h(t)=$ $\{(x(\tau), a(\tau)) \mid \tau=0,1,2, \ldots, t\}$, a realization of the random process from the "birth" time $t=0$, where $x(\tau)$ is the numerical sensory vector acquired at time $\tau$, and $a(\tau)$ is the numerical control vector of effectors at time $\tau$.

The history includes all of the information available to the agent from the "birth" time, such as the auditory sensations and the status of the agent's own actions.

Definition 2: The last context at time $t$ is the recent part of history, $l(t)=\{(x(\tau), a(\tau)) \mid \tau=t-b(t), \ldots, t-1, t\}$.

The last context consists of a short segment of recent experiences of temporal length $b(t)+1$. Currently in our work, $b(t)$ does not change with $t$ and different $b(t)$ are designed for different sensors and levels.

Definition 3: A state $s(t)$ is the internal representation of the context in an agent's internal information environment.

A state is a manipulated context generated by an agent.

According to the above definitions, as illustrated in Fig. 1, an agent views the world as a general random process, called history. The recent part of history is a last context. The state gives a more compact representation than a simple concatenation of the last contexts. Due to sensory uncertainty and partial observation, the state is also a random vector. The state at time instance $t$ depends on both the last context $l(t)$ and the last state $s(t-1)$. Therefore, we can recursively generate the state with

$$
s(t)=f(s(t-1), l(t))
$$




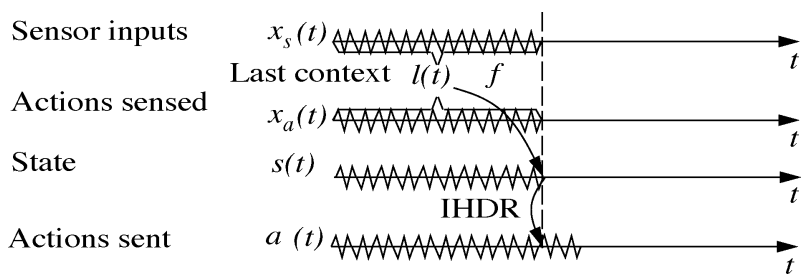

Fig. 1. Temporal illustration of the agent model. The last context includes information from sensors and the status of the agent's own actions. Depending on both the last context and the last state, the mapping $f$ returns a new state, associated with a list of actions. The action with the highest value is selected, which contains the effector control signals.

where the function $f$ generates states using feature derivation, temporal chucking, and vector quantization. The recursive estimation process can be modeled by a Markov decision process (MDP), where the state transition follows a conditional distribution

$$
P\left(s(t)=s \mid s(t-1)=s^{\prime}, l(t)=l\right)
$$

and the representation of $s(t)$ here is a high-dimensional vector. The most probable state at time $t$ is given by

$$
s^{*}(t)=\arg \max _{s \in S} P\left(s(t)=s \mid s(t-1)=s^{\prime}, l(t)=l\right)
$$

where $S$ is the set of all possible states, which is infinite. In a practical implementation, we approximate the set $S$ by an finite number of prototype states. These prototype states are incrementally merged cluster centers of many experienced state vectors.

The behavior of the agent is determined through a value system with respect to the states. To evaluate the value $Q$ of each action $a$ at each state $s$, a $Q$-value function $q(s, a)$ realizes a mapping $q: S \times A \rightarrow R$, where $A$ is the action space and $R$ is an one-dimensional (1-D) real coordinate space. The innate value system is such that the agent executes the action that has the highest estimated $Q$ value.

Fig. 2 gives a "snapshot" of the process running inside the agent. Driven by the last context $l(t)$, the agent transits from the current state $s(t)$ to the next state and sends an action vector $a_{p}(t)$ to the effectors. At the same time, the whole state set evolves by generating new states and merging existing states "on-the-fly." While Fig. 2 shows only five states, the total number of states can be hundreds or even more. Inside each state, a function is maintained to model the relationship between the $Q$-value and the action.

The previous way of modeling a decision process is called observation-driven Markov model (ODMM) [20], [21]. Different from the Markov models in common use, such as HMMs, the time dependence of the states in ODMM is conditioned explicitly upon past values instead of upon an unobservable underlying process. In summary, the major difference of the work reported here from a traditional MDP includes the following:

1) continuous high-dimensional vector space representation of states;

2) dynamically generated states;

3) a huge number of states with sparse state transition trajectories;

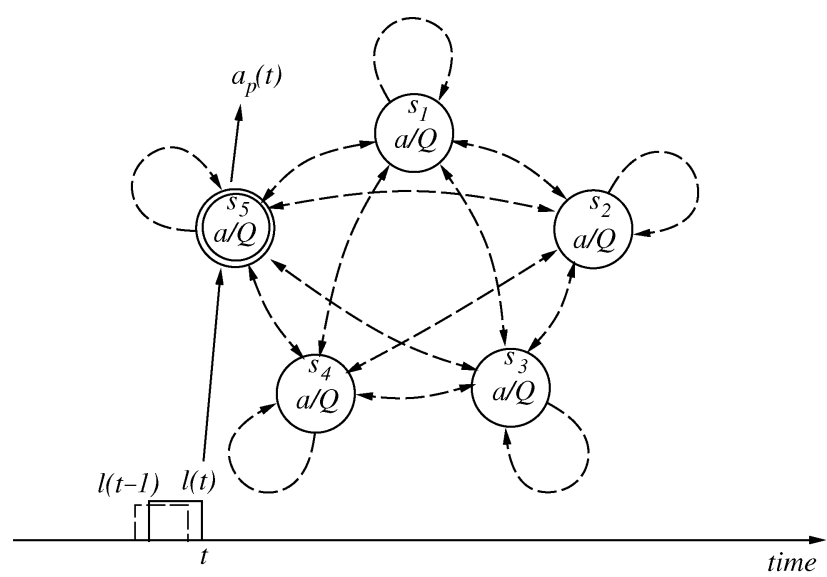

Fig. 2. Observation-driven MDP with dynamically generated states, context-driven state representation, and value-based action generation.

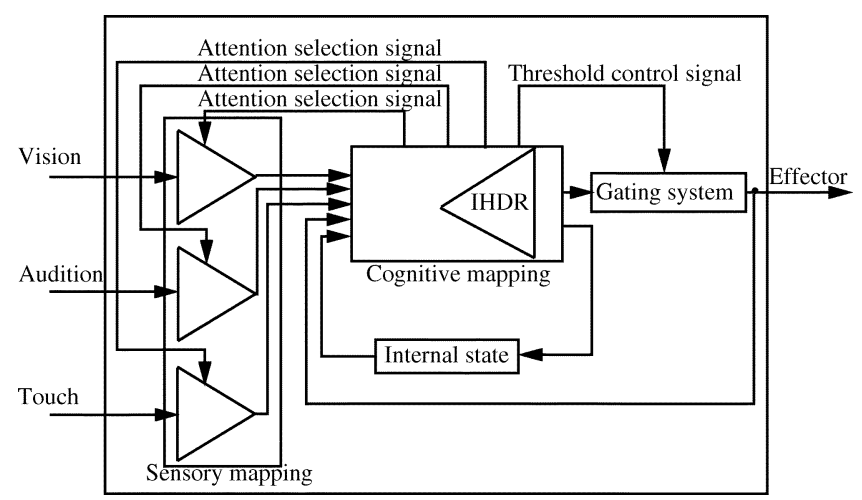

Fig. 3. Basic architecture of SAIL.

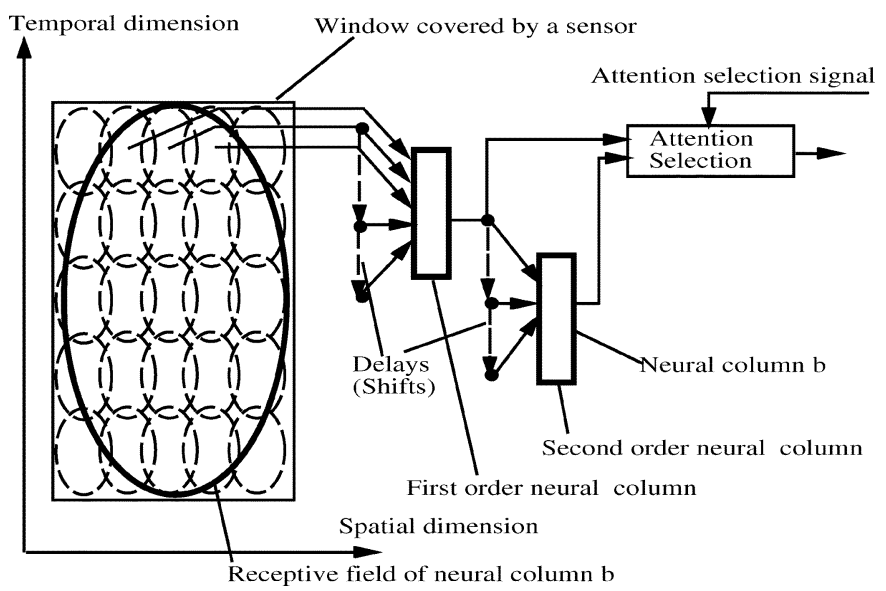

Fig. 4. Detailed architecture of the sensory mapping module.

4) using hierarchical discriminant regression (HDR) to incrementally estimate the observation-driven state transition;

5) using local temporal trajectory to estimate predicted $Q$-value.

The architecture that implements the model in Fig. 2 is shown in Fig. 3. Inputs from each sensor first enter a module called sensory mapping, which consists of a bank of developed filters. As shown in Fig. 4, considering the spatial and temporal expanse of the sensory inputs, we treat a sensor as a window moving 
in a spatiotemporal space with only the information inside the window getting through. We divide such a window into overlapped regions of different sizes, named receptive fields, each of which corresponds to an artificial neural column. These neural columns are organized in a hierarchical manner. The lower the layer is, the smaller the receptive fields of the neural columns are. The lowest layer is called the primitive layer and indexed as the 0-layer, whose neural columns cover the smallest receptive fields. The outputs from multiple neural columns in the $(i-1)$ th layer are used as the input to the neural columns in the $i$ th layer. Depending on whether the lower-layer neural columns spread along the spatial dimension or the temporal dimension, the neural columns in the higher layer may cover either wider field of view or a longer period of time. Suppose an $i$ th layer neural column cover $n_{i s} \times n_{i t}(i-1)$ th layer neural columns, where $n_{i s}$ and $n_{i t}$ represent the number of neural columns spreading along the spatial dimension and the temporal dimension, respectively, and the size of the primitive receptive field (the zeroth layer) is $l_{s} \times l_{t}$, where $l_{s}$ and $l_{t}$ are the spreads over the spatial dimension and the temporal dimension, respectively. Careful thinking will reveal that the size of the receptive field of an $i$ th layer neural column is

$$
\left[\Sigma_{k=1}^{i} n_{k s}-(i-1)\right] \times\left[\Sigma_{k=1}^{i} n_{k t}-(i-1)\right] \times l_{s} \times l_{t} .
$$

A neural column has two roles, developing a filter and computing the response using the filter updated so far. The role of development is carried out mainly by a technique called IPCA to compute the principal directions, along which the data population has the largest variations. The response is computed by the inner product between the input vector and the principal component vectors. With PCA, the responses from the neural columns of a lower layer keep most of the information while reducing the dimensionality of the inputs to a higher layer, which makes it easier for the neural columns in the higher layer to develop their own filters. The responses from the neural columns are fed into the next module, the cognitive mapping, after being selected by the control signals from the cognitive mapping. Since each neural column covers information within different receptive field, this selection is an internal behavior that enables the selective attention mechanism to handle the unsegmented sensory stream.

The cognitive mapping module is the central part of the system. It is responsible for learning the association between the sensory information from the environment and the behaviors that the system is supposed to produce. The behaviors can be both external and internal. External behaviors correspond to generating control signals for external effectors such as a joint motor of a robot arm, or any peripherals that the system is equipped with to act on the environment. Internal effectors include the previously mentioned attention selection effector in the sensory mapping module, the effector that manipulates internal states, and the threshold control effector in the gating system in Fig. 3.

Mathematically, we have what is called observation-driven state transition function $f$

$$
f: S \times L \rightarrow S
$$

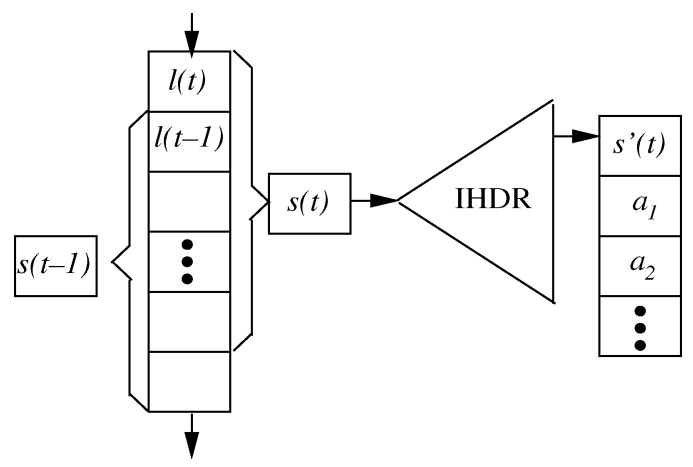

Fig. 5. Detailed architecture of the cognitive mapping module.

and the action generation function $g$

$$
g: S \times L \rightarrow 2^{A}
$$

where $S$ is the (local) state space, $L$ is the context space, $A$ is the output action space, and $2^{A}$ denotes the power set of $A$ (all the possible subsets of $A$ ). In other words, the cognitive mapping accepts the last state $s(t-1)$ and the current context $l(t)$ to generate a set of action candidates. The value system implements an action selection function $v$

$$
v: 2^{A} \rightarrow A
$$

according to the value of every action in the candidate action set.

In the real world, the number of all possible contexts is infinite. However, the resource of an agent is finite and so is the number of the states maintained by the agent. To resolve this conflict, we implemented an incremental hierarchical discriminant regression (IHDR) tree. The IHDR tree behaves as a vector quantizor (VQ) in the sense that it generates a set of codes or prototypes. In effect, the IHDR tree does much more than a traditional VQ by enforcing a discriminant regression mechanism and utilizing a hierarchical architecture. IHDR derives the features that are most relevant to the outputs and disregards the irrelevant ones. The tree structure keeps growing and merging nodes and, therefore, changes the prototype set constantly. In addition, the tree structure ensures to find the prototype that best matches the input in a fast logarithmic time for real-time speed. Associated with each prototype is a list of candidate actions as well as their expected values. The candidate that has the highest expected value is selected to be executed. In summary, IHDR implements both the $f$ and $g$ mappings at the same time. In practice, the above process was implemented as illustrated in Fig. 5. The last context $l(t)$ at time instance $t$ enters the state $s(t-1)$, which is represented by a first-in first-out queue, and updates it to the new state $s(t) . s(t)$ then updates and retrieves the IHDR tree to obtain a primitive prototype of the state $s^{\prime}(t)$, associated with a list of action vectors and their values.

The gating system is a part of the value system. It evaluates whether the intended action accumulates sufficient thrust to be issued as an actual action. In the implementation reported in this paper, the gating system simply provides a preset threshold. An action is taken only if its value, suggested by the cognitive mapping module, exceeds the threshold. 
In the following, we describe the major techniques used in the system development in details.

\section{A. IPCA}

We have discussed that each neural column in the sensory mapping module performs PCA. PCA is a well-known technique in data compression and feature extraction. It gives a linear transform that converts a set of $d$-dimensional data into a lower-dimensional space by minimizing the error in the least mean square (LMS) sense. Therefore, PCA is used to generate the new representation from sensory inputs.

A well-known approach to PCA is to solve an eigensystem problem. Given $A$ as the sample covariance matrix of the data set, one can find its eigenvectors and eigenvalues, sort the eigenvalues in descending order, and construct a $k \times d$ matrix $T$ with the rows being the eigenvectors corresponding to the largest $k$ eigenvalues. The matrix $T$ is the transform we are seeking [22]. This is the basic idea behind most techniques for PCA, such as the $Q R$ method [23]. However, since this approach requires the estimate of the covariance matrix, the data set usually needs to be completely available at the computation time. This is not appropriate for a developmental robot because of two reasons. First, a developmental robot is an online agent, which senses and responds to the environment continuously. It should not wait until all data is accumulated before doing the processing. Second, when the dimension of the data is high, both the computation and storage complexity grow dramatically. For example, in the eigenface method [24], [25], one of the promising face recognition methods that involves PCA, a moderate grey level image is of 88 rows and 64 columns, which results in a 5632-dimensional vector. Since the sample covariance matrix of a data set of $d$-dimensional random vectors contains $d(d+1) / 2$ independent numbers, this amounts to 15862528 numbers!

We proposed an algorithm, called candid covariance-free incremental PCA (CCIPCA), to incrementally compute the principal components of sequentially arriving samples without estimating the covariance matrix (so covariance-free). Suppose that sample vectors are acquired sequentially, $u(0), u(1), \ldots$, possibly infinite. Each $u(n), n=0,1, \ldots$, is a $d$-dimensional vector and $d$ can be as large as 5000 or even beyond. The $d \times d$ covariance matrix is neither known nor affordable to be estimated and stored as an intermediate result. Without loss of generality, we can assume that $u(n)$ has a zero mean. After the $n$th new sample $u_{n}$ is collected, the CCIPCA algorithm estimates the first $k$ dominant eigenvectors of the sample covariance matrix as follows:

$$
\begin{aligned}
v_{i}(n) & =\frac{n-1}{n} v_{i}(n-1)+\frac{1}{n} u_{i}(n) u_{i}^{T}(n) \frac{v_{i}(n-1)}{\left\|v_{i}(n-1)\right\|} \\
u_{i+1}(n) & =u_{i}(n)-u_{i}^{T}(n) \frac{v_{i}(n)}{\left\|v_{i}(n)\right\|} \frac{v_{i}(n)}{\left\|v_{i}(n)\right\|}
\end{aligned}
$$

where $i=1,2, \ldots, k$ is the index of the dominant eigenvectors, $v_{i}(n)$ is the $i$ th dominant eigenvector at time instance $n$. $u_{i+1}(n)$ is the residual vector at time instance $n$, i.e., the remaining vector after the first $i$ dominant components are subtracted from the sample $u(n)$. In particular, $u_{1}(n)$ is the residual vector after 0 dominant components is subtracted from the sample $u(n)$, which implies $u_{1}(n)=u(n)$.

The idea underlying CCIPCA comes from the concept of statistical efficiency of an estimate [26, p. 467].

Definition 4: An unbiased estimate $\hat{Q}$ of the parameter $Q$ is the most efficient estimate for the class $D$ of distribution functions if for every distribution function $f(u, Q)$ of $D$ the variance $D^{2}(\hat{Q})$ has the minimal value given by

$$
\begin{aligned}
D^{2}(\hat{Q}) & =E\left[(\hat{Q}-Q)^{2}\right] \\
& \geq \frac{1}{n \int_{-\infty}^{+\infty}\left[\frac{d \log f(u, Q)}{\partial Q}\right]^{2} f(u, Q) d u} .
\end{aligned}
$$

The inequality (7) is called Cramér-Rao inequality [27]. Basically, this definition says that the most efficient estimate is one that has the least variance from the real parameter and the variance is bounded by the Cramér-Rao inequality. For example, the sample mean, $\bar{x}=(1 / n) \sum_{i=1}^{n} x_{i}$, is the most efficient estimate for the mean of a Gaussian distribution with known standard deviation $\sigma$ [26].

For CCIPCA, by substituting itself recursively, (5) can be written as

$$
v_{i}(n)=\frac{1}{n} \sum_{j=1}^{n} u_{i}(j) u_{i}^{T}(j) \frac{v_{i}(j-1)}{\left\|v_{i}(j-1)\right\|} .
$$

Thus, $v_{i}(n)$ can be viewed as the mean of "samples" $w_{i}(j)$

$$
v_{i}(n)=\frac{1}{n} \sum_{j=1}^{n} w_{i}(j)
$$

where $w_{i}(j)=u_{i}(j) u_{i}^{T}(j)\left(v_{i}(j-1) /\left\|v_{i}(j-1)\right\|\right)$. Although $w_{i}(j)$ is not necessarily drawn from a Gaussian distribution independently, the estimate $v_{i}(n)$ seems to have a close relation with the estimate of a sample mean which is the most efficient estimate. We have proved that $v_{i}(n) \rightarrow \pm \lambda_{i} e_{i}$ with probability one when $n \rightarrow \infty$, where $\lambda_{i}$ is the $i$ th largest eigenvalue of the covariance matrix of $\{u(n)\}$ and $e_{i}$ is the corresponding eigenvector [28].

The previous analysis prompts a further improvement to CCIPCA. As shown in (9), all the "samples" $\left(\left\{w_{i}(j)\right\}\right)$ are weighted uniformly when $v_{i}(n)$ is estimated. Since $v_{i}(j)$ is far away from its real value at early estimation stage, the so-generated $w_{i}(j)$ can be regarded as a "sample" with large "noise" when $j$ is small. To help the convergence of the estimation, it is preferable to give smaller weight on these early "samples." A way to implement this idea is to use an amnesic average by changing (5) into

$$
v_{i}(n)=\frac{n-1-w}{n} v_{i}(n-1)+\frac{1+w}{n} u_{i}(n) u_{i}^{T}(n) \frac{v_{i}(n-1)}{\left\|v_{i}(n-1)\right\|}
$$

where $w$ is called amnesic parameter, typically, ranging from 2 to 4 . With $w,(10)$ automatically adjusts the weights of old estimates and new samples.

There were some earlier works on IPCA techniques. One of them was done by Oja and Karhunen [29], [30], who introduced 
an algorithm called stochastic gradient ascent (SGA). The major estimation steps of SGA are

$$
\begin{aligned}
\tilde{v}_{i}(n)= & v_{i}(n-1)+\gamma_{i}(n) u(n) u^{T}(n) v_{i}(n-1) \\
v_{i}(n)= & \text { orthonormalize } \tilde{v}_{i}(n) \text { w.r.t. } v_{j}(n), \\
& j=1,2, \ldots, i-1
\end{aligned}
$$

where, $v_{i}(n)$ is the $i$ th dominant eigenvectors of the samples' covariance matrix, $A=E\left\{u(n) u^{T}(n)\right\}, \tilde{v}_{i}(n)$ is the new estimate, and $\gamma_{i}(n)$ is a stochastic approximation gain. In practice, the orthonormalization can be done by a standard Gram-Schmidt Orthonormalization (GSO) procedure. The convergence of SGA was proved under some assumptions of $A$ and $\gamma_{i}(n)$ [30].

One of the major problems associated with SGA is the choice of $\gamma_{i}(n)$, which may be interpreted as the learning rate. Oja gave some suggestions on $\gamma_{i}(n)$ in [29], which is typically $1 / n$ multiplied by some constants. However, in practice, $\gamma_{i}(n)$ depends very much on the nature of the data and usually requires a trial-and-error procedure based on actual data. This is an efficiency issue. As we discussed previously, motivated by the concept of statistical efficiency of an estimate, CCIPCA was designed to handle this issue. More specifically, compared with SGA, CCIPCA does not do normalization on the estimate of eigenvectors [the first term on the right side of (5)], which leads us to the estimate of $\lambda e$ instead of $e$. Without doing normalization, the energy of the data is implicitly taken into account in the learning algorithm and no learning rate needs to be explicitly selected w.r.t. the data. This is the reason that we name this algorithm a candid IPCA algorithm. Some recent IPCA works introduce the inverse of eigenvalue estimates into the learning rate [31], [32]. The underlying idea is also to consider the energy of the data when updating the estimate of the eigenvectors so as to achieve adaptive learning. Better convergence rate than SGA has been demonstrated. Not being motivated by the concept of statistical efficiency, these algorithms keep the coefficient of the first term of (5) to be 1 , which might be the reason that they still require the learning rate to be experimentally found in order to ensure both fast convergence and stability. Further investigations, both theoretical and empirical ones, are yet to be conducted to compare these IPCA algorithms.

Another problem associated with SGA is that, as we can see from (11), the orthogonality between $v_{i}(n), i=1,2, \ldots$, is typically destroyed every time a new estimation is conducted. Except for the estimate of the first eigenvector, a GSO process is needed to recover the orthogonality, which is usually time-consuming. CCIPCA solves this problem by finding the first eigenvector and then subtracting the first component from the samples before computing the next component. Since each eigenvector is estimated in a subspace complementary to the subspace spanned by the principal components corresponding to larger eigenvalues, the orthogonality between the estimated principal components is enforced intrinsically. This idea was also used by other algorithms, such as Sanger's GHA [33].

In summary, the CCIPCA algorithm is as shown in Fig. 6. Although no mathematical analysis on the convergence rate is available yet, CCIPCA has been shown empirically to be a very
For $n=1,2, \ldots$, do,

1. $u_{1}(n)=u(n)$.

2. For $i=1,2, \ldots, k$, do,

2.1. If $i=n$, initialize the $i$ th eigenvector, $v_{i}(n-1)=u_{i}(n)$.

2.2. If $i<=n$, $v_{i}(n)=\frac{n-1-l}{n} v_{i}(n-1)+\underset{1+l}{n} u_{i}(n) u_{i}^{T}(n) \frac{v_{i}(n-1)}{\left\|v_{i}(n-1)\right\|}$, $u_{i+1}(n)=u_{i}(n)-u_{i}^{T}(n) \frac{v_{i}(n)}{\left\|v_{i}(n)\right\| \frac{v_{i}(n)}{\left\|v_{i}(n)\right\|}}$.

2.3. If $i>n$, initialize the $i$ th eigenvector, $v_{i}(n)=0$.

Fig. 6. CCIPCA algorithm: compute first $k$ dominant eigenvectors, $v_{1}(n), v_{2}(n), \ldots, v_{k}(n)$, directly from $u(n)$, where $n=1,2, \ldots$.

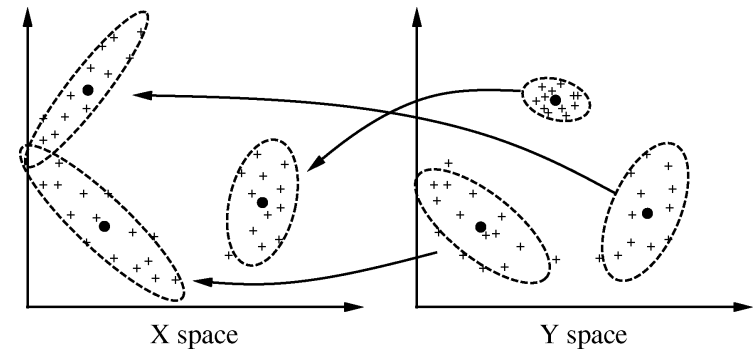

Fig. 7. Double clustering in both input and output spaces

efficient estimation algorithm compared with SGA and GHA for high-dimensional data [34].

\section{B. $I H D R$}

HDR is a new hierarchical statistical modeling method introduced by Hwang and Weng [35]. While we discuss the basic idea of HDR here, the reader is referred to the original paper for details.

Consider a general regression problem: approximating a mapping $h: X \rightarrow Y$ from a set of training samples $\left\{\left(x_{i}, y_{i}\right) \mid x_{i} \in X, y_{i} \in Y, i=1,2, \ldots, n\right\}$. HDR employs the idea of linear discriminant analysis (LDA) [36] to find such a mapping. LDA uses the class information and seeks an optimal linear transformation from the original data space to a new space in which the samples are well separated (or discriminated). In a typical usage of LDA, one needs to estimate the between-class and within-class scatter matrices, which requires the class information. However, for a regression problem, $y_{i}$ is a numerical vector and there are very few samples sharing a single $y_{i}$. In other words, there are very few samples in each class. Therefore, the within-class scatter matrix will be poorly estimated, especially for high-dimensional input data, e.g., a few thousand dimensions in vision problems or a few hundred dimensions in audition problems. HDR handles this issue with a coarse-to-fine way.

First of all, $q$ y-clusters in the output space are generated according to the output part of the training samples using a k-means-like algorithm. The training samples is, thus, gathered into $q$ super groups. The x-part of the samples is accordingly clustered and we call these clusters $x$-clusters (see Fig. 7). Since $q$ is typically a small number, e.g., 5 , it is effective to estimate the between-class and within-class scatter matrices for these $q$ $\mathrm{x}$-clusters. We call this process a double clustering process. The 


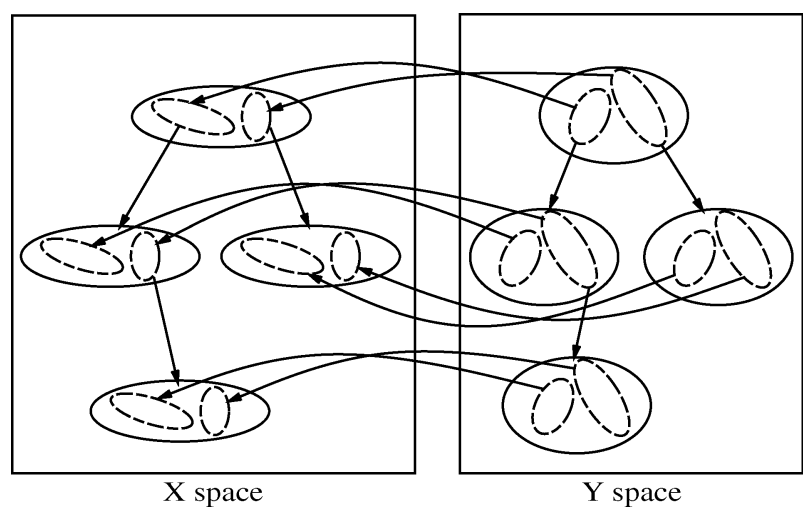

Fig. 8. Hierarchical discriminant analysis.

$q$ X-cluster centers span a $(q-1)$-dimensional space, which we call a discriminant space because it characterizes the discriminant information among the x-clusters. A probability-based distance in the discriminant space is used to determine which $\mathrm{x}$-cluster a test sample belongs to.

As we may immediately realize, while the double-clustering process is done on the whole training set, it can be recursively used for the data in each of the super groups (see Fig. 8), which ends up with a tree structure. Moreover, the deeper nodes in the tree, the smaller variance the data has. After the tree is constructed, we may generate the output of a new input $x$ by retrieving the tree. The final output $y$ would be the mean of the $\mathrm{y}$-cluster of the leaf node on the retrieval path.

In summary, HDR has two major advantages. First, it automatically generates representations by finding the discriminant spaces according to the events (data) encountered. These subspaces are optimal for different particular tasks in terms of discriminant capability. Second, the hierarchical structure not only reduces the computational complexity of searching but also enables the system to make use of more samples at shallow levels where the variance of the data is large, which is critical to parameter estimation. At the deep levels, the sample variance is tremendously reduced and correct decisions can be made with very few number of samples. Because of the first advantage, HDR has little limitation in terms of representation power and potentially can fit any data. Because of the second advantage, HDR can learn without any iterations. As a result, HDR realizes one-instance learning without local minima, i.e., zero error on training data without iterations.

HDR has been successfully used in face recognition [35]. In that application, we have a training data set ready for the computation of statistics. However, a developmental robot conducts online learning, which means that the data is only available sequentially. In this case, the previous HDR method needs to be modified so as to be used incrementally (IHDR) [15].

A major difficulty of IHDR implementation is that incremental computation of the statistics, which includes the sample means and scatter matrices of both x-clusters and y-clusters, may result in bad estimates of cluster boundary. At the beginning of the incremental procedure, because the number of samples is small, the clusters are usually ill-generated. So is the cluster boundary, which will influence later partitions of the tree. In IHDR, we use the amnesic average technique to gradually get rid of the effects of earlier data.

Suppose $\left\{u_{i}, i=1,2, \ldots, n\right\}$ are the data entering the system sequentially. Written in an incremental style, their sample mean, $\bar{u}$, and scatter matrix, $\Gamma_{u}$ are given by

$$
\begin{aligned}
\bar{u}^{(n+1)} & =\frac{n \bar{u}^{(n)}+u_{n+1}}{n+1} \\
& =\frac{n}{n+1} \bar{u}^{(n)}+\frac{1}{n+1} u_{n+1} \\
\Gamma_{u}^{(n+1)} & =\frac{n}{n+1} \Gamma_{u}^{(n)}+\frac{1}{n+1}\left(u_{i}-\bar{u}\right)\left(u_{i}-\bar{u}\right)^{T} .
\end{aligned}
$$

The incremental estimation of the statistics is done by treating each sample's contribution equally with weight $1 / n$. As time elapses, $n$ increases and a new sample would make less and less difference on the estimation. An amnesic averaging mechanism is implemented in the following way:

$$
\begin{aligned}
\bar{u}^{(n+1)} & =\frac{n-l}{n+1} \bar{u}^{(n)}+\frac{1+w}{n+1} u_{n+1} \\
\Gamma_{u}^{(n+1)} & =\frac{n-l}{n+1} \Gamma_{u}^{(n)}+\frac{1+w}{n+1}\left(u_{i}-\bar{u}\right)\left(u_{i}-\bar{u}\right)^{T}
\end{aligned}
$$

The amnesic parameter $w$ changes the relative weighting of the old estimate and the new sample. By setting $w$ larger than 0 , new samples will have more effects and old samples are "forgotten" gradually.

\section{Generation of External Behaviors}

The nature of autonomous development of our system prevents us from defining the behaviors in advance. While this offers flexibility in the ultimate behavior capability of the system, it immediately introduces a problem: how to acquire the desired actions among a very large number of possible actions?

Recalling the body design step of the autonomous mental development paradigm in Section I, we know that the external action space is determined at the system design time. For example, if the robot arm is made, its joint space is determined too, although not all points in this space are reachable. Therefore, the system can generate all possible external actions by accumulating a small and fixed set of internal action increments. For example, to touch every $(x, y)$ position in a two-dimensional space, a minimum of four increment vectors are sufficient: $(1,0)$, $(-1,0),(0,1),(0,-1)$.

\section{Unified Learning Strategy}

Now we are ready to discuss the learning process of the developmental robot. In general, there exist three learning strategies, supervised learning, reinforcement learning, and unsupervised learning. As we believe, in terms of learning behavior, no system can learn effectively without any supervision, we leave out unsupervised learning and only discuss the first two here.

Supervised learning enables a mapping from the input space to the output space using a set of given input-output pairs. This learning mode is efficient when the continuous presence of input-output pairs is available, e.g., a vision-guided vehicle 
learns control when a human teacher holds the steering wheel during training.

There are situations when supervised learning is not practical. For example, a robot needs internal behaviors such as selecting receptive fields. A trainer does not have access to these internal actions and may only give reward or punishment according to the robot's external behaviors to encourage or discourage the internal behaviors. This needs a reinforcement learning process.

$Q$-learning is one of the most popular reinforcement learning algorithms. $Q$-learning and its variance have been widely used in action selection [37]-[40]. In $Q$-learning, each state maintains a set of action values, called $Q$-values. The action with the largest value will be selected as the system output. The $Q$-learning algorithm is as follows:

$$
\begin{aligned}
Q(s(t-1), a(t)) \leftarrow(1-\alpha) Q(s(t-1), a(t)) & \\
& +\alpha\left[r(t)+\gamma \max _{a^{\prime}} Q\left(s(t), a^{\prime}\right)\right]
\end{aligned}
$$

where $a$ and $a^{\prime}$ are actions associated with a state, $s(t-1)$ is the state at time $t-1, s(t)$ is the state that the system lands on after executing action $a(t), r(t)$ is the reward received from the environment, $\alpha$ is the learning rate, and $\gamma$ is the time-discount rate. The algorithm shows that $Q$-values are updated according to the immediate reward $r(t)$ and the value of the next state, which allows delayed reward to be back-propagated in time during learning. After enough experiences of trial-and-error, the system may predict the reward correctly when similar contexts occurs the next time. Particularly, $\alpha$ scales the correction term $\left(r(t)+\gamma \max _{a^{\prime}} Q\left(s(t), a^{\prime}\right)-Q(s(t-1), a(t))\right.$ that updates the old value $Q(s(t-1), a(t))$. It should be relatively small so that the learning can converge, although a too small $\alpha$ may trap $Q$ to a local minimum. We used 0.1 in practice. $\gamma$ specifies how far in the history the current reward will be back-propagated. The closer to 1 it is, the farther the reward will be back-propagated. We used 0.9 in practice. A detailed discussion on $\alpha$ and $\gamma$ can be found in [38].

We have adapted $Q$-learning to integrate supervised learning and reinforcement learning strategies into a unified mode (Fig. 9). ${ }^{2}$ In our integrated learning mode, if no action is imposed by the environment, we follow the $Q$-learning algorithm to update the action values. If an action is imposed by the environment, we increase the value of the corresponding action with a fixed amount. Typically, we make this amount large so as to reflect the fact that the system should follow the instructions of the trainer. There is not a traditional concept of learning rate in the learning process because the system does not learn a fixed representation, e.g., a vector. Instead, the knowledge representation is a tree that keeps changing, i.e., growing or merging leaves. The changes take place when necessary. For example, when an auditory stimulus enters the IHDR tree, if there has been a primitive prototype very similar to the new stimulus, the new auditory stimulus will be discarded. On the other hand, if the closest primitive prototype is still totally different from the stimulus, the new auditory stimulus will be

${ }^{2}$ Related work on integrating reinforcement and supervised learning can be found in [41].

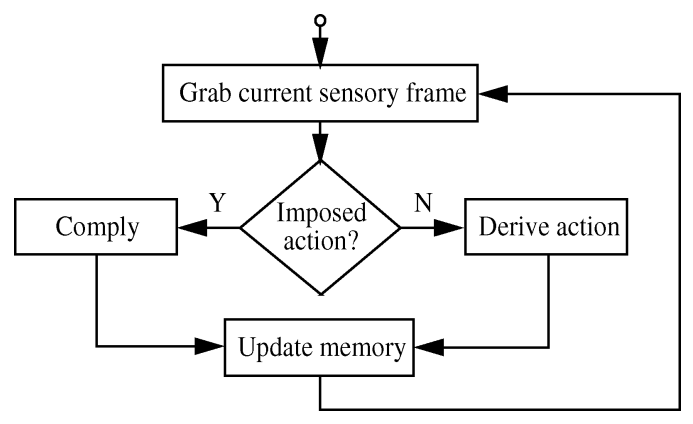

Fig. 9. Unified learning strategy.

internalized to be a new primitive prototype in the IHDR tree. If we really want to discuss the process with respect to a learning rate, the learning rate is zero in the first case and is one in the second case. In this sense, the learning rate is adaptive to the experience of the robot. The complete learning process in each learning cycle is shown in Fig. 10.

In the learning process, an important parameter is the temporal span of the last context, i.e., how far in the past the agent looks to identify the state. A long span is desirable in order to keep the important causal information. Although a too long span may also degrade the performance by introducing unrelated signals or noise, both the sensory mapping and the cognitive mapping are meant for selecting task-related information and, therefore, addressing this issue. In the experiments presented here, our criterion is to extend the span as long as the system can keep up with the real-time performance.

We also predetermined the maximum number of clusters in each node of the IHDR tree. In general, a larger number produces better performance, although it reduces the speed of the algorithm. In the experiments presented in this paper, we set it to be five. This is again a tradeoff between the real-time requirement and the performance.

An online developmental system does not explicitly have separated training and testing sessions. Therefore, the learning process is repeated at all time instances. In the following discussions, when we mention a testing session, we simply mean "freezing" any changes to the system so that we may evaluate it.

In a traditional reinforcement machine learning application, the machine usually has a particular goal, for example, winning in a chess game. A significant reward is usually given at the "end" time of the task showing whether the system reaches the goal or not. However, in the case of a developmental robot, there is no fixed goal and the "end" time will be the robot "death" time. During the lifetime of the robot, there may be multiple tasks (e.g., following different commands) at a time, and the tasks may change as the time passes by. Therefore, instead of giving significant rewards by the end of the robot life, we shape the behavior of the robot by giving significant reward at the end of every interactive cycle. In the following experiments, an interactive cycle corresponds to an uttered command followed by a desired action. This way of using reinforcement learning is motivated by animal learning such as in the case of parenting or animal training. 
1. Grab a new auditory frame $x(t)$.

2. Update each neural column of the sensory mapping module by doing PCA incrementally.

3. Concatenate the outputs from the neural columns in the sensory mapping module and the current external action vector, $a(t)$, into a single vector, $l(t)$. Suppress the outputs from some of the neural columns to zero according to the attention selection signals (part of action $a(t)$ ).

4. Replace the oldest part of the internal state $s(t-1)$ with $l(t)$ to get a vector $s(t)$.

5. Query the IHDR tree and get a primitive prototype, $s^{\prime}(t)$, that is closest to $s(t)$ using the fast tree search.

6. If $s(t)$ is significantly different from $s^{\prime}(t)$, it is considered as a new sample and we update the IHDR tree by saving $s(t)$. Otherwise, $s(t)$ updates $s^{\prime}(t)$ through incremental averaging.

7. After updating the tree, we query it again using $s(t)$ to get the primitive prototype $s^{\prime}(t)$.

8. If action $a(t)$ is given (imposed by a trainer), increase the value of the action that is most similar to $a(t)$ and return $a(t)$ as the new action at this time instance.

9. Otherwise, update $Q$-values of $s(t-1)$ using Eq. (17) and return $\arg \max _{a^{\prime}} Q\left(s(t), a^{\prime}\right)$ as the new action at this time instance.

10. If the value of the new action passes the threshold set by the gating system, the action is released in the form of an external action vector to the effectors. Otherwise, the external action vector is set to be zero, which means no real action.

11. Return to step 1.

Fig. 10. Robot learning process in each execution cycle.

\section{E. Communicative Learning}

While both supervised learning and reinforcement learning have some advantages and they compensate each other in our unified learning strategy, they are relatively low-level learning mechanisms and demand a lot of training efforts in complex behavior learning. For example, to teach a robot to "go left at the corner," the human trainer has to either push the robot to turn (supervised learning) or give some rewards when the robot happens to turn left at the corner (reinforcement learning). Actually, in animal learning and human learning, there is a higher level of learning mode, which we call communicative learning. Communicative learning takes the advantage of a higher animal's capability of language acquisition to transfer sophisticated skills from the trainer to the learner through language instructions. In the case of the previous example, suppose "recognizing a corner" and "following a 'turn left' command" are two learned behaviors. Teaching "go left at the corner" would be as easy as saying "turn left" when the robot reaches the corner. As a preliminary investigation of communicative learning, we taught the robot to learn vision-guided navigation after it acquired some simple language capability and the results are reported in Section IV-D.

\section{EXPERIMENTAL RESULTS}

\section{A. Experiments on Audition-Driven Behavior Development with Reinforcement Learning}

The first experiment was to train a system to develop the audition-driven behaviors through reinforcement learning.

To examine the behavior, especially those internal actions, of the system in detail, we first conducted a simulation study in which the speech data were read from files and then fed into the system continuously exactly as what would happen in real time. In the follow-up real-time study, the prerecorded utterances were played one after the other with about 1-s silence in between. In both the simulation and the real-time cases, the speech data appeared to the system simply as a continuous auditory stream without any marks indicating the starting or ending points of the utterances.

The speech data was collected as follows. 140 persons with a variety of nationalities (including American, Chinese, French, Indian, Malaysian, and Spanish) and ages (from 18 to 50) participated in our speech data collection. Each person made five utterances for each of the four vowels, /A/(hard), /E/(head), $/ \mathrm{i} /\left(\right.$ heed) and $/ \mathrm{c} /(\mathrm{haul})^{3}$ The sound was digitized at 11.025 $\mathrm{kHz}$. In this way, we got an speech data set with 2800 isolated utterances.

The speech data was fed into the system continuously in both training and testing sessions. 13-order Mel-frequency Cepstral Coefficients (MFCC [42]) were computed over every 256 sound sample points (about $20 \mathrm{~ms}$ ) before the data reached the sensory mapping module. The sensory mapping module had four layers. The neural columns in each layer took as the input the outputs from ten neural columns of the next lower layer, spreading along the temporal dimension. Referring to (4) and considering the temporal dimension only, the layers from the lowest to the highest cover $10 \times 20=200 \mathrm{~ms},(10+10-1) \times 20=380 \mathrm{~ms}$, $(10+10+10-2) \times 20=560 \mathrm{~ms},(10+10+10+10-4) \times 20=$ $720 \mathrm{~ms}$, respectively.

Each layer ran the CCIPCA algorithm to develop the filters. The experiment was arranged so that the higher layer did not start training until the lower layer become mature or, in other words, until the eigenvectors in the lower layer converged. While the reader is referred to [34] for a detailed discussion on the performance of CCIPCA, Fig. 11 shows the performance of CCIPCA at the first layer. Particularly, the coherence between the normalized eigenvector $v$ estimated by CCIPCA and the one $v^{\prime}$ computed by batch method is represented by their inner

\footnotetext{
bets.
} 

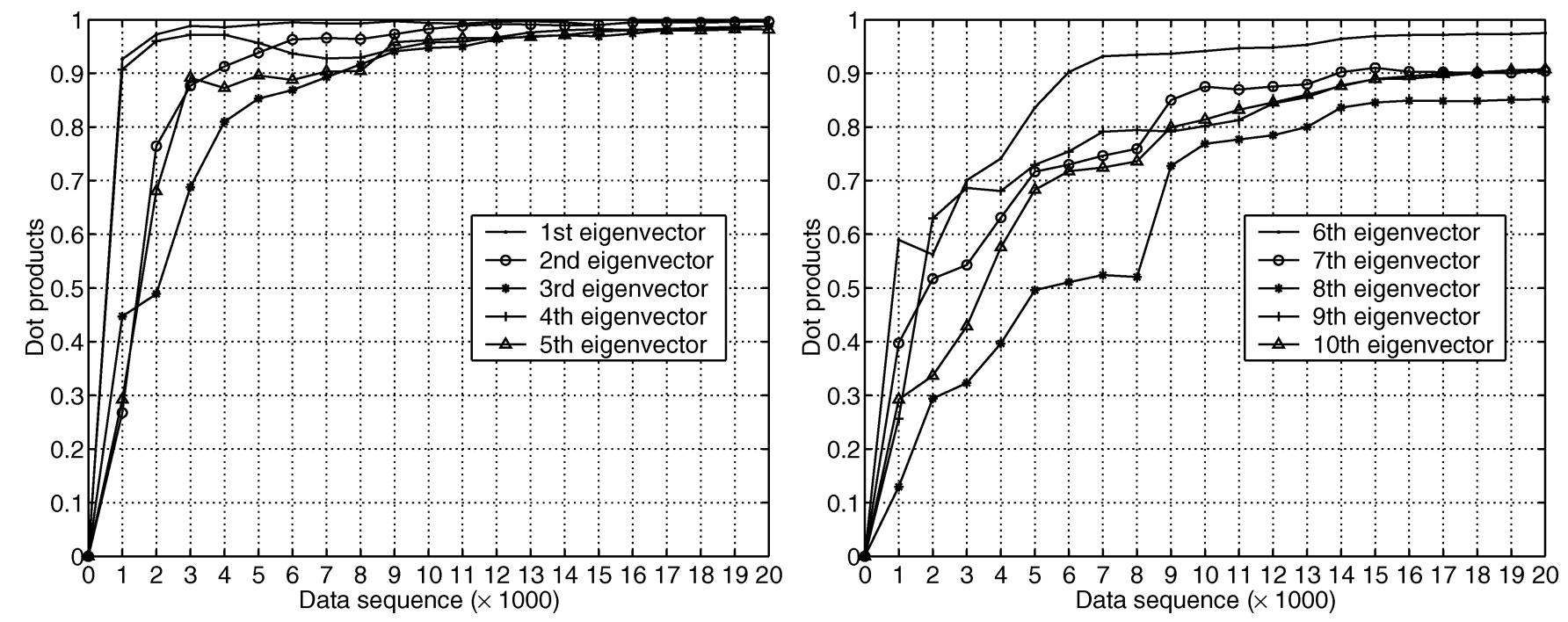

Fig. 11. Coherence of the first 10 eigenvectors computed by CCIPCA with the ones obtained by batch PCA.

TABLE I

DiMENSIONALITY OF THE DATA INTO AND OUT OF EACH SENSORY MAPPING LAYER

\begin{tabular}{c|c|c|c|c}
\hline Layer no. & 1 & 2 & 3 & 4 \\
\hline Input dim & 130 & 310 & 270 & 220 \\
Output dim & 31 & 27 & 22 & 18 \\
\hline
\end{tabular}

product $v \cdot v^{\prime}$. Because $\left\|v-v^{\prime}\right\|=2\left(1-v \cdot v^{\prime}\right)$, we have $v=v^{\prime}$ iff $v \cdot v^{\prime}=1$. In other words, the closer to 1 the inner product is, the better the coherence of the two vectors is. As we can see from Fig. 11, the eigenvectors obtained by CCIPCA converged very well after training was conducted upon about 7 -min auditory data $(20,000 * 20 \mathrm{~ms}=400 \mathrm{~s})$.

To determine the total number of eigenvectors, a criterion was set to keep $95 \%$ of variance of the data. The actual percentage of the kept variance was estimated by the ratio between the summation of the first $n$ eigenvalues and the variance of the data. The reduction of dimensionality in each layer is summarized in Table I.

The effector of the system was represented by a four-dimensional (4-D) action vector. Four desired behaviors were defined, each for one of the four vowels. Behaviors were identified by the component of the action vector with the maximum value. For example, if the first component of the action vector had the maximum value, it was identified as action 1. Four internal actions were defined, each doing an increment on one component of the action vector. There was an extra internal action that reset the whole action vector to zero. Each state had all these internal actions when the state was created. The initial value of the "reset" internal action was 0.1. The initial values of other internal actions were 0 . Since the "reset" internal action had a larger initial value, it was selected first when the system entered a newly created state and, thus, reset the action vector. This is desirable because the system should not do anything when it is not sure what is preferable to do.

The rewards were decided as follows. If the system made an action within a range of $(-200 \mathrm{~ms}, 200 \mathrm{~ms})$ at the end of an utterance and the action was correct, the system received a reward

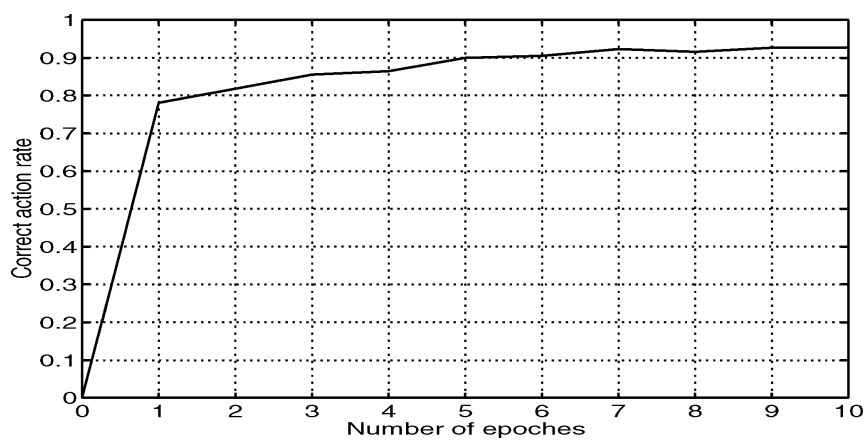

Fig. 12. Simulation results: correct action rate versus epoches.

TABLE II

SiMULATION RESUlTS: CONFUSION TABLE

\begin{tabular}{c|c|c|c|c|c}
\hline E.B. vs. A.B. & $\mathrm{a}$ & $\mathrm{e}$ & $\mathrm{i}$ & $\mathrm{o}$ & Rejection \\
\hline $\mathrm{a}$ & 125 & 3 & 0 & 5 & 7 \\
$\mathrm{e}$ & 1 & 127 & 9 & 0 & 3 \\
$\mathrm{i}$ & 0 & 5 & 134 & 0 & 1 \\
$\mathrm{o}$ & 5 & 0 & 0 & 133 & 2 \\
\hline
\end{tabular}

1. If the action was wrong or there was no action made within that time window, the system got a reward -1 . In all other cases including the silence period, the system got reward -0.001 .

The performance was evaluated as follows. Within a short period before or after the end of an utterance, if the system reacted correctly once or more than once within that time window, we counted it as a success. Otherwise it is an error. The test was done using five-fold cross-validation.

1) Simulation: In the simulation experiment, we fed the speech data set into the system repeatedly. Each repetition of the data feeding is called an epoch. The result in Fig. 12 shows that the system gradually improved its behavior through practice. The confusion table of the expected behaviors (E.B.) versus the actual behaviors (A.B.) is shown in Table II.

To take a closer look at the behavior of the system, we traced one of the primitive prototypes in the IHDR tree that corre- 


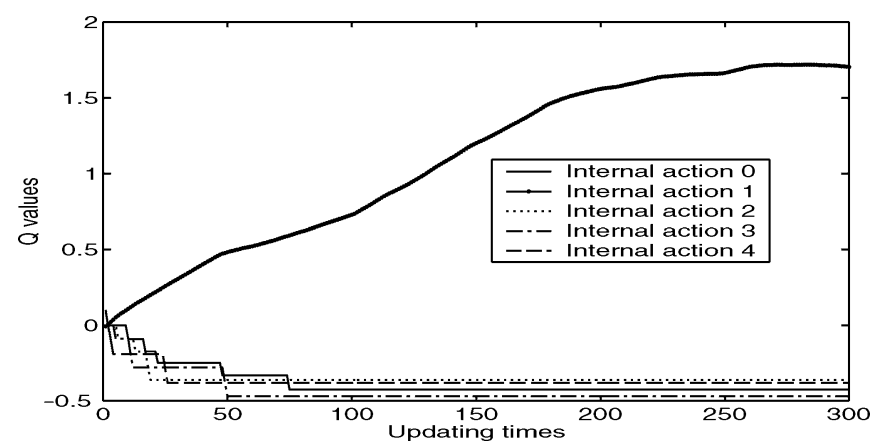

Fig. 13. Simulation results: smoothed $Q$ values of the internal actions of one of the states versus updating times.

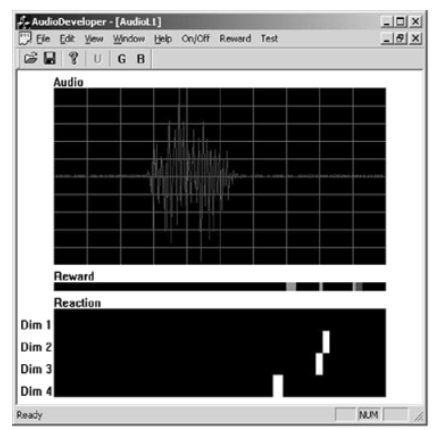

(a)

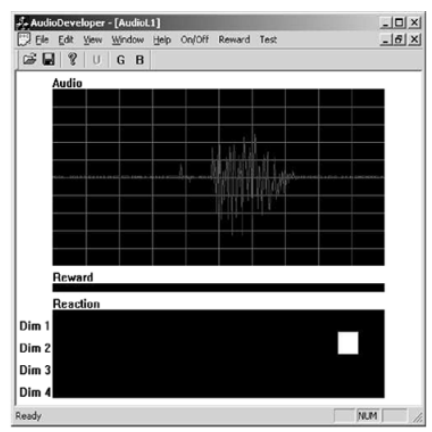

(b)
Fig. 14. GUI of AudioDeveloper. (a) During online learning. (b) After online learning.

sponded to vowel /A/. In the beginning, when this primitive prototype was created, the $Q$-values of all the internal actions were zero except for internal action 0 , the "reset" internal action. Therefore, the action vector of the system was zero and the system did not do anything. This external behavior resulted in the negative rewards from the trainer and the $Q$-value of internal action 0 was reduced. When it became lower than zero, other internal actions competed to take over according to their $Q$-values. If several of them had the same $Q$-values, one of them was selected randomly. If the selected one was internal action 1 , the first component of the action vector would be increased, which ended up with the desired external action and a positive reward would be given to the system. As a result, the $Q$-value of internal action 1 would be increased. If other internal actions were selected, undesirable external actions would be taken and the system would receive negative rewards. As a result, the $Q$-value of the corresponding internal action would be reduced. The actual changing process of the $Q$-values was shown in Fig. 13, which suggested that the system gradually and reliably figured out that it should always select to pick up internal action 1 and execute the desirable external action, when hearing vowel /A/.

2) A Real-Time System: To test the system in a more realistic situation, we built a software agent called AudioDeveloper. AudioDeveloper had two kinds of sensors, a microphone as the auditory sensor and two touch sensors as reward sensors. A simulated four-joint robot arm was the effector. As shown in Fig. 14, the graphical user interface (GUI) of AudioDeveloper displays the auditory wave (the top part) and the actions (the bottom part) along the horizontal time axis while the rewards is shown in

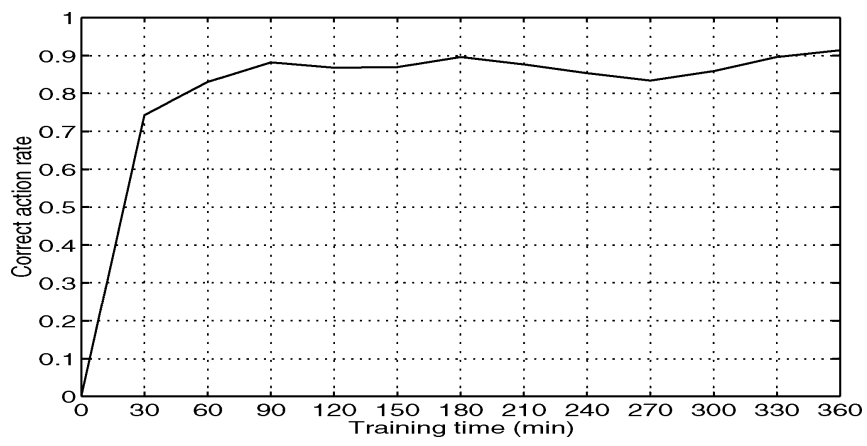

Fig. 15. Performance of AudioDevelopmer: correct action rate versus training time.

TABLE III

Frequency of the Attention Selection Behavior Per EXTERNAL BEHAVIOR

\begin{tabular}{c|c|c|c|c|c|c|c|c|c|c}
\hline External Behavior & 1 & 2 & 3 & 4 & 5 & 6 & 7 & 8 & 9 & 10 \\
\hline 1st layer & 0 & 1.00 & 0.73 & 0.83 & 0.66 & 0.74 & 0 & 0.63 & 0.25 & 0.40 \\
2nd layer & 1.00 & 0 & 0.27 & 0.17 & 0.33 & 0.26 & 1.00 & 0.38 & 0.75 & 0.60 \\
\hline
\end{tabular}

the middle part of the GUI. Human trainers first spoke to AudioDeveloper and then gave the rewards by pushing the " $G$ " and "B" buttons on the GUI toolbar for "good" and "bad," respectively, according to AudioDeveloper's behavior. To facilitate the training procedure, another software agent, AudioTeacher, was programmed as the real-time virtual teacher. AudioTeacher played the prerecorded sound, and generated and sent the reward to AudioDeveloper according to AudioDeveloper's response.

The training procedure was run in real-time for $6 \mathrm{~h}$, during which the resulting developed system was saved every half an hour so that we could examine the performance later. Fig. 15 shows that the correct action rate of the system increased quite steadily as the time passed by.

\section{B. Experiments on Selective Attention Learning}

In the experiments presented in Section IV-A, the attention selection signals were set in advance so that the outputs from all sensory mapping layers were selected.

To specifically test the system's attention selection capability, we collected speech data of 10 numbers (1-10) which had more context information than vowel data. The simulation experiments were conducted in a way similar to the ones in last section. The difference was that the sensory mapping module here had only two layers and that the system had one more internal action to select the output from one of the two layers. After being trained for 10 epoches, the correction rate of the external actions were improved from $0 \%$ to $95.2 \%$. We examined the system's choices of attention at the time the system made the external behaviors. In Table III, the first line shows the external behaviors represented by their corresponding number utterances. The second and third lines show the frequency that the system selected the first or second layer when firing external behaviors.

An interesting thing happened to external behaviors 1 and 7 as we expected. The system exclusively chose the second layer when making decisions. After a second thought, one will realize that the tail parts of the utterances of "one" and "seven" 

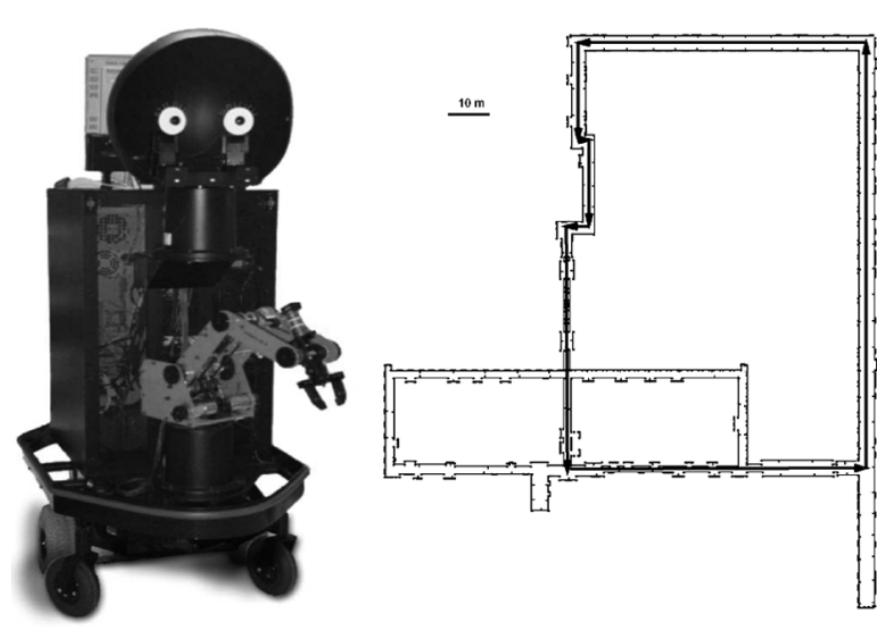

(a)

(b)

Fig. 16. (a) SAIL robot at Michigan State University. (b) Second floor of the Engineering Building at Michigan State University.

are similar. Therefore, the first layer did not cover enough context for decision making and the system decided to choose the second layer. For "nine" and "ten," which had similar tail parts, the system also showed its preference by selecting the second layer. In other words, the attention selection behavior was successfully acquired.

\section{Experiments on the Sail Robot}

In the following experiments, we trained the SAIL robot to act correctly according to real-time auditory inputs.

SAIL [Fig. 16(a)] is a human-size mobile robot house-made at Michigan State University. It has a drive-base, a six-joint robot arm, a rotary neck, and two pan-tilt units, on which two charged-coupled device (CCD) cameras (as eyes) are mounted. A wireless microphone functions as an ear. SAIL has four pressure sensors on its torso and 28 touch sensors on its eyes, arm, neck, and bumper. Its main computer is a high-end dual-processor dual-bus PC workstation with 512-MB RAM and a $27-G B$ three-drive disk array. All the sensory information processing, memory recall and update as well as real-time effector controls are done in real-time.

The SAIL robot was taught to follow verbal commands by moving its body, arm, and eyes. The 15 verbal commands were "go left," "go right," "forward," "backward," "freeze," "arm left," "arm right," "arm up," "arm down," "hand open," "hand close," "see left," "see right," "see up," and "see down."

The training process was conducted online in real-time through physical interactions between a trainer and the SAIL robot. It consisted of two phases, supervised learning followed by reinforcement learning. In the supervised learning phase, a trainer spoke to the robot with a spoken command $C$ and then imposed a desired action $A$ by pressing a pressure sensor or a touch sensor that was linked to the corresponding effector. In reinforcement learning, a "good" button and a "bad" button were used to simulate appetizing and aversive sensors, respectively. The reward was decided in the same way as in the experiments presented in Section IV-A. We still set the SAIL
TABLE IV

PERFormance of THE SAIL Robot IN ONE-TRAINER CASE

\begin{tabular}{c|c|c|c|c|c}
\hline Commands & Go left & Go right & Forward & Backward & Freeze \\
\hline Total times & 35 & 23 & 65 & 7 & 5 \\
Correct rate(\%) & 97.1 & 91.3 & 93.8 & 100 & 80 \\
\hline Commands & Arm left & Arm right & Arm up & Arm down & Hand open \\
\hline Total times & 10 & 10 & 10 & 10 & 10 \\
Correct rate(\%) & 100 & 90 & 100 & 100 & 90 \\
\hline Commands & Hand close & See left & See right & See up & See down \\
\hline Total times & 10 & 10 & 10 & 10 & 10 \\
Correct rate(\%) & 90 & 100 & 100 & 100 & 100 \\
\hline
\end{tabular}

TABLE V

PERFORMANCE OF THE SAIL ROBOT WHEN FOLLOWING THE 12TH TRAINER's COMMAND IN MULTITRAINER CASE

\begin{tabular}{c|c|c|c|c|c}
\hline Commands & Go left & Go right & Forward & Backward & Freeze \\
\hline Total times & 36 & 28 & 70 & 8 & 9 \\
Correct rate(\%) & 88.9 & 89.3 & 92.8 & 87.5 & 88.9 \\
\hline Commands & Arm left & Arm right & Arm up & Arm down & Hand open \\
\hline Total times & 10 & 10 & 10 & 10 & 10 \\
Correct rate(\%) & 90 & 90 & 100 & 100 & 90 \\
\hline Commands & Hand close & See left & See right & See up & See down \\
\hline Total times & 10 & 10 & 10 & 10 & 10 \\
Correct rate(\%) & 80 & 100 & 100 & 100 & 100 \\
\hline
\end{tabular}

robot a default attention selection behavior to select all sensory mapping layers.

After being trained for $15 \mathrm{~min}$, the SAIL robot could follow commands with about a $90 \%$ correct rate. Table IV summarizes the performance of the SAIL robot when it was guided by the verbal commands to navigate through the corridors of the Engineering Building at MSU [see Fig. 16(b)]. The arm and eye commands were issued 10 times each at different locations.

To further test the system's capability of dealing with speaker variations, we conducted a multitrainer experiment. Eleven persons participated in training. They spoke each of the 15 commands for five times which resulted in 825 utterances. The speech data (four out of five utterances of each commands) was fed into the SAIL robot offline appended with appropriated actions at the end of each utterance. The SAIL robot with partially trained "brain" started to run in real-time. Then, a trainer, being the 12th trainer, taught the SAIL robot through physical interactions four times for each command. In this way, we simulated the situation that a partially developed SAIL robot continuously developed its audition-driven behaviors.

After training, the 12th trainer tested the SAIL robot by guiding it through the second floor of the Engineering Building, just as was done in the one-trainer case. The performance is summarized in Table V. More trainers introduced more variance in speech. The results show that the performance of the SAIL robot in the multitrainer case degraded a little compared with the one-trainer case but it was still reasonable. The performance for other trainers was evaluated offline using the left-out utterances. The performance is summarized in Table VI. The shape of the IHDR trees after trained by one trainer and by twelve trainers are shown in Fig. 17, where the horizontal axis is the depth of the tree and the vertical axis is the number of nodes. 

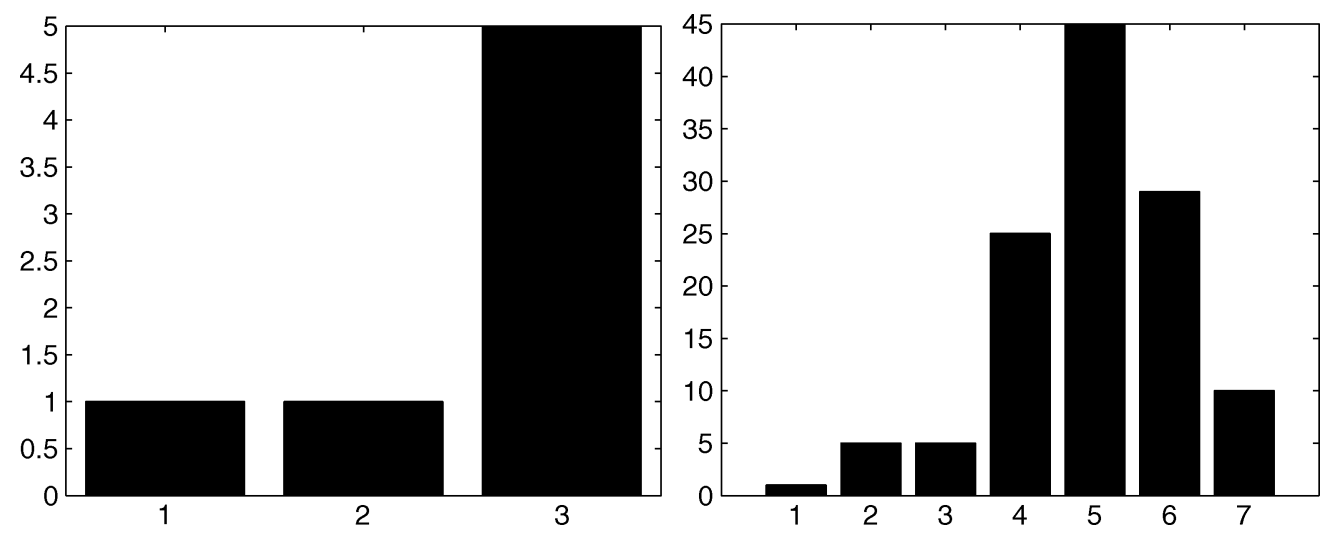

Fig. 17. SAIL robot: the shape of IHDR tree after trained by one trainer and twelve trainers. The horizontal axis specifies the depth of the tree and the vertical axis

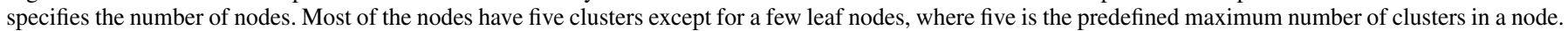
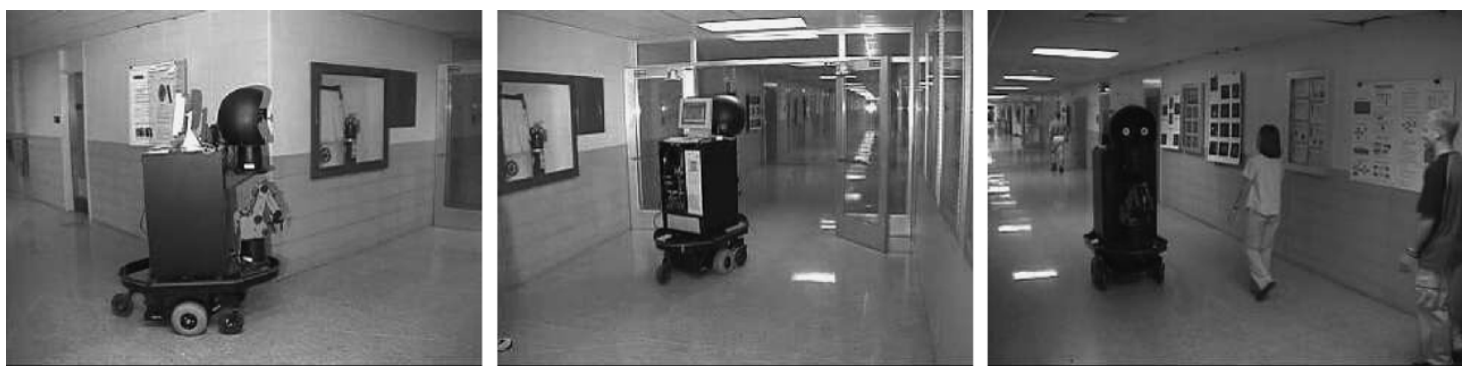

Fig. 18. SAIL robot is running autonomously in the hallway of the Engineering Building of Michigan State University.

TABLE VI

PERFORMANCE OF THE SAIL ROBOT ON OFF-LiNE TEST DATA IN Multitrainer CASE

\begin{tabular}{c|c|c|c|c|c}
\hline Commands & Go left & Go right & Forward & Backward & Freeze \\
\hline Correct rate(\%) & 94.5 & 89.9 & 92.7 & 100.0 & 100.0 \\
\hline Commands & Arm left & Arm right & Arm up & Arm down & Hand open \\
\hline Correct rate(\%) & 100.0 & 90.9 & 96.3 & 92.7 & 89.9 \\
\hline Commands & Hand close & See left & See right & See up & See down \\
\hline Correct rate(\%) & 89.9 & 90.9 & 92.7 & 100.0 & 100.0 \\
\hline
\end{tabular}

While the maximum number of clusters in each node was predetermined to be five, the total number of clusters in a whole IHDR tree is much more than a few, which is understandable by referring back to Fig. 2. When the window of last context slips along the time axis, the number of states (clusters) formed by the sensory information entering the cognitive mapping is typically more than the number of voice commands which was fifteen in the real robot experiment. Multiple trainers generated even more states (a few hundreds) due to the variance of their voices.

At this point, the reader may have better sense about the difference between developmental learning and other existing robot learning. Specifically, in the previous experiments, the auditory learning is conducted by the robot based upon continuous and unlabeled speech streams without any prior knowledge about the auditory signals, such as the designated language or the phoneme models. Further, prior knowledge about the action to be performed is not available to the system either. The robot develops its audition-driven behaviors through physical interactions with the environment. This is the first work, as far as we know, on online speech learning without a predesigned model and with the number of words and the number of speakers totally open. ${ }^{4}$ Although the performance described previously has not matched those of the traditional SR systems in term of vocabulary, this work has made a solid progress for the very difficult new learning mode that simulates human/animal learning.

\section{Experiments on Communicative Learning}

The previous learning experiments are in fact a process of simple language acquisition with rich semantics and little syntax. ${ }^{5}$ The semantics is naturally acquired here because the speech is learned within the physical context. Upon learning to follow verbal commands, the SAIL robot was taught to perform vision-guided navigation through communicative learning.

Communicative learning includes two phases. The first phase is language acquisition, which has been achieved previously. The second phase is teaching using language (Fig. 18). Using the trained commands, the trainer guided and instructed the robot to go through the entire second floor of the Engineering Building at Michigan State University. The actions executed under the corresponding voice command were associated with the visual context by another IHDR tree in real time. Fig. 19 shows some of the images seen by the SAIL robot. Later, when the robot saw a similar visual context, it would retrieve the IHDR mapping and find the practiced action. In the experiment presented here, the switch of the attention between vision and audition was an innate (programmed-in) volume-based behavior of the robot. The voice command had a higher priority

\footnotetext{
${ }^{4}$ The work in [4] was for recognizing five vowels in a simulated online mode. ${ }^{5}$ It is typically the case for a child.
} 

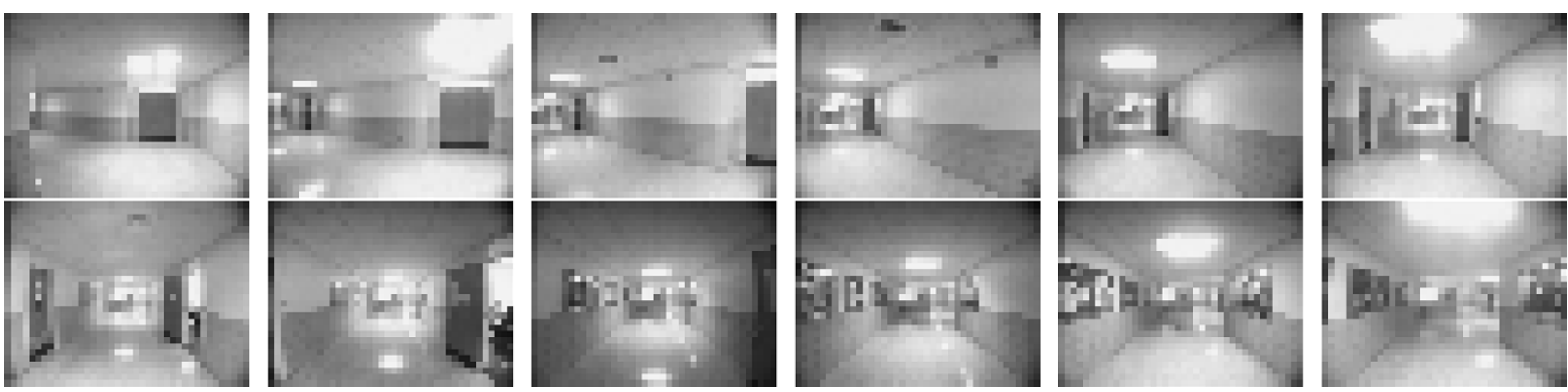

Fig. 19. Examples of the image sequence seen by the SAIL robot

to control the robot than the learned vision-guided controls. After training, the SAIL robot was tested for 10 turns in the Engineering Building until the robot batteries ran low. It needed further voice instructions at only five locations which were difficult for vision-guided navigation because of the changing lighting (windows) or very narrow corners.

The experiment of communicative learning reported here saved the trainer a lot of effort in training by using verbal commands. Behavior scaling-up using more complex languages is a subject of our further studies.

\section{CONCLUSION}

We have demonstrated that it is feasible for an artificial system to develop its audition-driven behaviors through online, real-time interaction with the environment. This work is among a few works that enable a machine to learn directly from unsegmented and unlabeled speech streams, a mode in which human children learn. IHDR played a great role in the success of the experiments. It automatically derives discriminant features and, thus, automatically generates internal representations. PCA was used to extract salient features and reduce the input dimensionality in the sensory mapping. A fast algorithm, CCIPCA, was developed to obtain the eigenvectors incrementally without computing the large covariance matrix. The behaviors of the robot were developed through a unified learning strategy. While external behaviors such as body movement were imposed through a supervised learning procedure, internal behaviors such as selective attention were acquired through autonomous learning, such as reinforcement learning. Our experiments show that after a short process of grounded simple language acquisition, the robot can produce desired behaviors upon hearing verbal commands. We have also showed that this simple language capability can be used later in communicative learning for other behavior learning, such as vision-guided navigation. In the future, we plan to teach the robot more complex behaviors and test its capability by keeping it running for a much longer time.

\section{REFERENCES}

[1] L. R. Rabiner, "Toward vision 2001: voice and audio processing considerations," AT\&T Tech. J., vol. 74, no. 2, pp. 4-13, 1995.

[2] Proc. NSF/DARPA Workshop Development and Learning, J. Weng and I. Stockman, Eds., East Lansing, MI, Apr. 5-7, 2000.

[3] J. Weng, J. McClelland, A. Pentland, O. Sporns, I. Stockman, M. Sur, and E. Thelen, "Autonomous mental development by robots and animals," Science, vol. 291, pp. 599-600, Jan. 26, 2001.
[4] J. Weng, Y. B. Lee, and C. H. Evans, "The developmental approach to multimedia speech learning," in Proc. IEEE Int. Conf. Acoustics, Speech, and Signal Processing, Phoenix, AZ, Mar. 15-19, 1999, pp. 3093-3096.

[5] S. E. Levinson and Q. Liu et al., "The role of sensorimotor function, associative memory and reinforcement learning in automatic acquisition of spoken language by an autonomous robot," in Proc. Workshop Development Learning, East Lansing, MI, Apr. 5-7, 2000.

[6] A. L. Gorin, D. Pertrovksa-Delacretaz, and G. Riccardi et al., "Learning spoken language without transcriptions," in Proc. Int. Workshop Automatic Speech Recognition and Understanding, CO, Dec. 12-15, 1999.

[7] J. Piaget, The Origins of Intelligence in Children. New York: International Universities, 1956.

[8] A. M. Leslie, "Some implications of pretense for mechanisms underlying the child's theory of mind," in Developing Theories of Mind, J. W. Astington, P. L. Harris, and D. R. Olson, Eds. Cambridge, U.K.: Cambridge Univ. Press, 1988, pp. 19-46.

[9] R. Baillargeon, "The object concept revisited: new directions in the investigation of infants' physical knowledge," in Visual Perception and Congition in Infancy, C. Granrud, Ed. Hillsdale, NJ: Lawrence Erlbaum, 1993, pp. 265-316.

[10] R. Held and A. Hein, "Movement-produced stimulation and the development of visualll guided behaviors," J. Comparative Physiol. Psychol., vol. 56, pp. 872-876, 1963.

[11] B. I. Bertenthal, J. J. Campos, and K. C. Barrett, "Self-produced locomotions: an organizer of emotional, cognitive, and social development in infancy," in Continuities and Discontinuities in Development, R. Emde and R. Harmon, Eds. New York: Plenum, 1984.

[12] H. V. B. Hirsch and D. N. Spinelli, "Modification of the distribution of receptive field orientation in cats by selective visual exposure during development," Exp. Brain Res., vol. 13, pp. 509-527, 1971.

[13] D. H. Hubel, T. N. Wiesel, and S. LeVay, "Plasticity of ocular dominance columns in monkey striate cortex," Philos. Trans. R. Soc. Lond., vol. 278 , pp. 377-409, 1977.

[14] R. Spitz, "Anaclitic depression," Psychoanal. Study of Child, vol. 2, pp. 313-342, 1946.

[15] J. Weng, W. S. Hwang, Y. Zhang, C. Yang, and R. Smith, "Developmental humanoids: humanoids that develop skills automatically," in Proc. 1st IEEE-RAS Int. Conf. Humanoid Robots, Boston, MA, Sep. 7-8, 2000.

[16] N. Almassy, G. M. Edelman, and O. Sporns, "Behavioral constraints in the development of neuronal properties: a cortical model embedded in a real-word device," Cerebral Cortex, vol. 8, pp. 346-361, Jun. 1998.

[17] Q. Liu, S. Levinson, Y. Wu, and T. Huang, "Robot speech learning via entropy guided LVQ and memory association," in Proc. INNS-IEEE Int. Joint Conf. Neural Networks, Washington, DC, Jul. 14-19, 2001, pp. 2176-2181.

[18] D. Roy, B. Schiele, and A. Pentland, "Learning audio-visual associations using mutual information," in Proc. Int. Conf. Computer Vision, Workshop on Integrating Speech and Image Understanding, Corfu, Greece, Sep. 1999.

[19] D. Roy, "Learning from multimodal observations," in Proc. IEEE Int Conf. Multimedia and Expo, New York, Jul. 2000, pp. 579-582.

[20] D. R. Cox, "Statistical analysis of time series: some recent developments," Scand. J. Statist., vol. 8, no. 2, pp. 93-115, 1981.

[21] S. L. Zeger and B. Qaqish, "Markov regression models for time series: a quasi-likelihood approach," Biometrics, vol. 44, no. 4, pp. 1019-1031, Dec. 1988.

[22] K. Fukunaga, Introduction to Statistical Pattern Recognition, 2nd ed. New York: Academic, 1990. 
[23] G. H. Golub and C. F. van Loan, Matrix Computations. Baltimore, MD: The Johns Hopkins Univ. Press, 1989.

[24] I. Sirovich and M. Kirby, "Low-dimensional procedure for the caracterization of human faces," J. Opt. Soc. Amer. A, vol. 4, no. 3, pp. 519-524, Mar. 1987.

[25] M. Turk and A. Pentland, "Eigenfaces for recognition," J. Cogn. Neurosci., vol. 3, no. 1, pp. 71-86, 1991.

[26] M. Fisz, Probability Theory and Mathematical Statistics, 3rd ed. New York: Wiley, 1963.

[27] C. R. Rao, Ed., Linear Statistical Inference and Its Applications, 2nd ed. New York: Wiley, 1973.

[28] Y. Zhang and J. Weng, "Convergence analysis of complementary candid incremental principal component analysis," Dept. Comput. Sci. Eng., Michigan State Univ., East Lansing, MI, Tech. Rep. MSU-CSE-01-23, Aug. 2001.

[29] E. Oja, Subspace Methods of Pattern Recognition. Letchworth, U.K.: Research Studies, 1983.

[30] E. Oja and J. Karhunen, "On stochastic approximation of the eigenvectors and eigenvalues of the expectation of a random matrix," J. Math. Anal. Appl., vol. 106, pp. 69-84, 1985.

[31] L.-H. Chen and S. Chang, "An adaptive learning algorithm for principal component analysis," IEEE Trans. Neural Netw., vol. 6, no. 5, pp. 1255-1263, Sep. 1995.

[32] R. Mller and A. Knies, "Coupled principal component analysis," IEEE Trans. Neural Netw., vol. 15, no. 1, pp. 214-222, Jan. 2004

[33] T. D. Sanger, "Optimal unsupervised learning in a single-layer linear feedforward neural network," IEEE Trans. Neural Netw., vol. 2, no. 6, pp. 459-473, Nov. 1989.

[34] J. Weng, Y. Zhang, and W. S. Hwang, "Candid covariance-free incremental principal component analysis," IEEE Trans. Pattern Anal. Mach. Intell., vol. 25, no. 8, pp. 1034-1040, Aug. 2003.

[35] W. Hwang and J. Weng, "Hierarchical discriminant regression," IEEE Trans. Pattern Anal. Mach. Intell., vol. 22, no. 11, pp. 1277-1293, Nov. 2000.

[36] R. O. Duda, P. E. Hart, and D. G. Stork, Pattern Classification, 2nd ed. New York: Wiley, 2001.

[37] C. J. Watkins, "Q-learning," Mach. Learn., vol. 8, pp. 279-292, 1992.

[38] R. S. Sutton and A. G. Barto, Reinforcement Learning-An Introduction. Cambridge, MA: MIT Press, 1998.

[39] A. J. Smith, "Applications of the self-organizing map to reinforcement learning," Neural Netw., vol. 15, pp. 1107-1124, 2002.

[40] K. Iwata, K. Ikeda, and H. Sakai, "A new criterion using information gain for action selection strategy in reinforcement learning," IEEE Trans. Neural Netw., vol. 15, no. 4, pp. 792-799, Jul. 2004.

[41] J. A. Clouse, "Learning from an automated training agent," in Adaptation and Learning in Multiagent Systems, G. Weis and S. Sen, Eds. Berlin, Germany: Springer-Verlag, 1996.

[42] J. R. Deller, J. G. Proakis, and J. H. L. Hansen, Discrete-Time Processing of Speech Signals. New York: Macmillan, 1993.

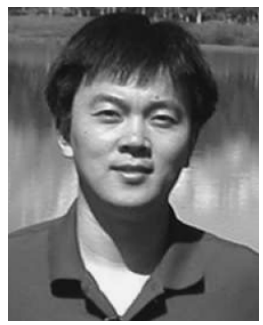

Yilu Zhang (S'99-M'02) received the B.S. and M.S. degrees in electrical engineering from Zhejiang University, Hangzhou, China, in 1994 and 1997, respectively, and the Ph.D. degree in computer science from Michigan State University, East Lansing, MI, in 2002 .

He joined the Research Center, General Motors Corporation, Warren, MI, in 2002 and currently holds a position of Senior Research Engineer. His research interests include human machine interactions, machine learning, speech processing, image processing, and multimedia technologies.

Dr. Zhang is a Member of the Society of Automotive Engineers (SAE), Sigma Xi International Honor Society, and the Autonomous Mental Development Technical Committee of the IEEE Computational Intelligence Society. He serves as an Associate Editor of International Journal of Humanoid Robotics, and the Editor of the Autonomous Mental Development Technical Committee Newsletter of the IEEE Computational Intelligence Society.

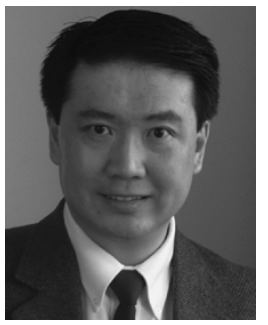

Juyang Weng (S'85-M'88) received the Ph.D. degree in computer science from the University of Illinois, Urbana, in 1989

He is a Professor in the Department of Computer Science and Engineering, Michigan State University, East Lansing, MI. His research interests include computer vision, speech recognition, human-machine multimodal interface using vision, audition, speech, gesture and actions, and intelligent robots. $\mathrm{He}$ is the author of over one hundred research articles and book chapters. He is a coauthor (with T. S. Huang and N. Ahuja) of the book Motion and Structure from Image Sequences (New York: Springer-Verlag, 1993). He is the Editor-in-Chief of the International Journal of Humanoid Robotics.

Dr. Weng is an Associate Editor for the IEEE TRANSACTIONS ON PATTERN RECOGNITION AND MACHINE INTELLIGENCE and, from 1994 to 1997, was an Associate Editor of the IEEE TRANSACtions on IMAge PROCESSING. He is the Chairman of the Autonomous Mental Development Technical Committee of the IEEE Computational Intelligence Society. He was a program Cochair of the 2000 NSF/DARPA Workshop on Development and Learning, held at Michigan State University and a Program Cochair of the IEEE 2nd International Conference on Development and Learning (ICDL'02), held at Massachusetts Institute of Technology, Cambridge, MA.

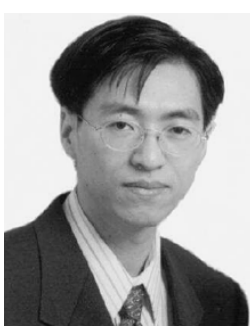

Wey-Shiuan Hwang received the B.S. degree in computer science from the National Taiwan University, Taipei, Taiwan, in 1990 and the M.S. and Ph.D. degrees in computer science from Michigan State University, East Lansing, MI, in 1995 and 1999, respectively.

From 2000 to 2001, he was a a Research Associate in the Department of Computer Science, Michigan State University. In 2001, he joined Rudolph Technologies Inc., Flanders, NJ, where he is currently a Pattern Recognition Engineer. His research interests include computer vision, autonomous learning robots, and pattern recognition. 